\title{
A Comparison of Physical Layers for Low Power Wide Area Networks
}

\author{
Yoann Roth ${ }^{1}$, Jean-Baptiste Doré ${ }^{1}$, Laurent Ros ${ }^{2}$, and Vincent Berg ${ }^{1}$ \\ ${ }^{1}$ CEA, LETI, MiNATEC Campus, F-38054 Grenoble, France \\ ${ }^{2}$ Univ. Grenoble Alpes, GIPSA-Lab, F-38000 Grenoble, France \\ \{yoann.roth, jean-baptiste.dore, vincent.berg\}@cea.fr \\ laurent.ros@gipsa-lab.grenoble-inp.fr
}

\begin{abstract}
In Low Power Wide Area networks, terminals are expected to be low cost, low power and able to achieve successful communication at long range. Communication should be low rate compared to cellular mobile networks. Most of the current technologies dedicated to Machine-toMachine communication rely on the use of a spreading factor to achieve low levels of sensitivity. To contain power consumption at the terminal side, the cost of complexity should be paid by the receiving side. We propose to use turbo processing schemes as potential physical layers. We compare these schemes to a standard and an industrial solution, and show that a significant gain in sensitivity can be achieved, and very energy efficient scheme can be designed by mixing turbo processing and orthogonal modulation concepts.
\end{abstract}

Key words: Low Power Wide Area $\cdot$ LPWA · Internet of Things · PHYlayer · FSK · Turbo Code · Low Rate · Low SNR · Machine-to-Machine

\section{Introduction}

The Internet-of-Things (IoT) is a vast concept, where every object is expected to be connected. Amongst the foreseeable applications for these Machine-toMachine (M2M) communications, many require the design of a new Low Power Wide Area (LPWA) network [1]. While recent advances (3G, 4G) sought for higher data rate and spectral efficiency, this kind of network is expected to be low rate, low power and long range. Packet sizes should be from a few bytes to hundreds maximum. Terminal power consumption and sensitivity working levels are critical issues, relying on the choice of an efficient and robust physical layer. We consider a star network topology, where each node is connected to a base-station. We focus on the uplink communication.

To achieve low sensitivity levels, a widely used technique is the low complexity repetition code. Indeed, repeating the information by a Spreading Factor (SF) $\lambda$ at the transmitter can offer a gain of $10 \log _{10}(\lambda) \mathrm{dB}$ in sensitivity at the receiver side. This technique offers a good compromise between sensitivity and complexity and many systems rely on it, as for instance in Direct Sequence Spread Sprectrum (DSSS). Another natural choice for low data rate and low 
power applications is the use of $M$-ary orthogonal modulation. Increasing the size of the alphabet gives a gain in energy efficiency, while reducing the spectral efficiency [2]. This property can be extended to Orthogonal Sequence Spread Spectrum (OSSS), and using high orders of alphabet can bring low levels of sensitivity.

Some solutions have been proposed to answer the need of a new LPWA network. A recent proposal $[3,4]$ is based on the combination of OSSS with a low complexity block code. Also, efforts have been made to design the standard 802.15.4k using the DSSS technique combined with a convolutional code [5], which can be considered for LPWA networks. These two schemes are low complexity and straightforward solutions. However, since low power consumption is expected mainly at the terminal side, the cost of complexity can be paid by the receiver side, i.e the base station. More elaborate decoding schemes can then be used, such as turbo processing [6], and lead to a better energy efficiency of the system. We consider the widely known Turbo Code (TC) standardized by the Universal Mobile Telecommunications System (UMTS) [7, 8] combined with a common modulation and a SF as a potential physical layer for LPWA. Our recent work [9] pointed out that if the repetition scheme allows a decrease of the required Signal-to-Noise Ratio (SNR) of a specific modulation, it does not increase the energy efficiency of the system (which can be expressed in terms of energy per bit to noise spectral density ratio, denoted $E_{b} / N_{0}$ ). This is a consequence of the information rate reduction implied by the use of the SF. Redundancy should be used more efficiently by the receiver, by means of turbo processing. The scheme proposed in [9], dedicated to LPWA networks, combines orthogonal modulation with turbo processing, and achieves very promising level of performance.

In this paper, we propose a comparison of four different schemes: the 802.15.4k standard [5], OSSS with a Hamming code, the TC UMTS from $[7,8]$ with a common modulation (Binary Phase Shift Keying (BPSK) or $M$-ary orthogonal modulation), and the Turbo-FSK scheme proposed in [9]. All of them perform at a low spectral efficiency, aim a high energy efficiency, and would be suitable for uplink communication in the context of LPWA networks. We focus on the physical layer, and assume perfect synchronization and Additive White Gaussian Noise (AWGN). All architectures are described, and simulations are performed. To the best of our knowledge, comparison of these potential LPWA networks have never been done. Also, the use of the UMTS Turbo Code and a modulation have never been considered before as a potential uplink scheme for LPWA.

The paper is organized as follows. The different schemes and their architectures are introduced Section 2. Performance comparison are presented Section 3. In Section 4, results are discussed and Section 5 concludes the paper. 


\section{System Model}

This section is dedicated to the description of the four conceivable physical layers previously mentioned. For each one, a short introduction is followed by the presentation of the block diagram of the transmitter. As receivers is rarely standardized, a receiver scheme has been proposed for each physical layer.

\subsection{IEEE 802.15.4k}

The IEEE $802.15 .4 \mathrm{k}$ is a standard for local and metropolitan area networks, and is part of the Low-Rate Wireless Personal Area Networks (LR-WPANs) [5]. It aims at low energy critical infrastructure monitoring networks. This standard supports three physical layer (PHY) modes: DSSS with BPSK or OffsetQuadrature Phase Shift Keying (O-QPSK), or Frequency Shift Keying (FSK). DSSS with BPSK modulation is adapted to more constrained situations, and will be presented here. Standard specifications allow the use of a spreading factor value $\lambda$ from 16 to 32768 .

Block diagrams for transmitter and receiver are given in Figure 1. The transmitter is composed of a Forward Error Correction (FEC) block, defined to be the convolutional code of rate $1 / 2$, with generators polynomial [171 133] (in octal) and constraint length $k=7$. After encoding, interleaving is done to ensure diversity at the reception side. Differential encoding is applied, and encoded data is "repeated" by the use of a binary direct sequence of size $\lambda$. BPSK modulation is then used to transmit the signal through the channel. The normalized spectral efficiency of the physical layer, expressed in bits/s/Hz, is

$$
\eta_{1}=\frac{1}{2 \lambda}
$$

The receiver executes the reverse operations of the transmitting side. After despreading the signal (executing the mean weighted by the elements of the binary direct sequence), we use a soft differential BPSK decoder. Knowing how data was interleaved at the transmitter side, the deinterleaving operation allows the soft input Viterbi decoder to decode the data and to finally retrieve the information bits.

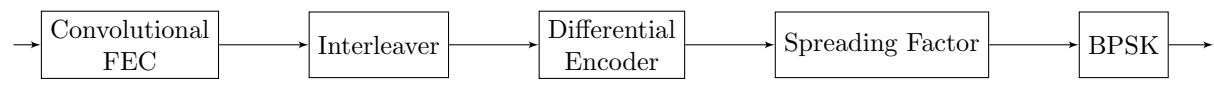

(a)

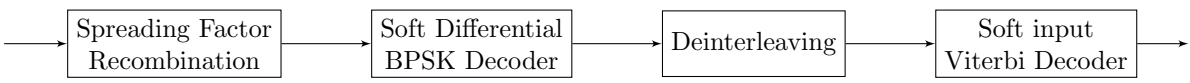

(b)

Fig. 1. Standard IEEE 802.15.4k: (a) transmitter architecture, (b) receiver architecture. 


\subsection{Orthogonal modulation with block code}

It is a known fact that orthogonal modulations reach channel capacity for large size of alphabet [2]. An original solution relies on the use of orthogonal modulations with a simple channel code $[4,10]$. Spreading operation is replaced by increasing the alphabet size, thus lowering the spectral efficiency but also increasing the energy efficiency. As we assumed ideal synchronization and AWGN channel, performance does not depend on the orthogonal alphabet choice $(e . g$ FSK, OSSS, Pulse Position Modulation, ... ).

The transmitter and receiver architectures are given in Figure 2. After applying a simple Hamming $(7,4,3)$ block code, coded bits are interleaved and then mapped to an orthogonal alphabet of size $\lambda$. The spectral efficiency is defined as

$$
\eta_{2}=\frac{\log _{2}(\lambda)}{\lambda} \frac{4}{7}
$$

At the receiving side, soft demodulation is performed. The output is the log likelihood ratio (LLR) of the bits. It is defined as

$$
L\left(b_{n}\right)=\log \frac{p\left(b_{n}=1\right)}{p\left(b_{n}=0\right)} .
$$

The principle of soft decoding is to compute the A Posteriori Probabilities (APP) of the codewords, i.e for each codeword the probability of having a given codeword knowing the observation, and then compute the LLR of the information bits, knowing the APP for all the possible codewords. The elements of the orthogonal alphabet are denoted $\boldsymbol{c}^{i}$ with $i \in[0, \lambda-1]$ and $\boldsymbol{c}^{i}=\left[\boldsymbol{c}_{0}^{i}, \ldots \boldsymbol{c}_{\lambda-1}^{i}\right]$. For each $\boldsymbol{c}^{i}$, the associated information word is denoted $\boldsymbol{b}^{i}=\left[\boldsymbol{b}_{0}^{i}, \ldots \boldsymbol{b}_{\log _{2}(\lambda)-1}^{i}\right.$. We consider one noisy received codeword $\boldsymbol{y}$, and $\boldsymbol{d}$ its associated decoded information word. The APP can be expressed as

$$
p\left(\boldsymbol{c}^{i} \mid \boldsymbol{y}\right)=\frac{p\left(\boldsymbol{y} \mid \boldsymbol{c}^{i}\right) p\left(\boldsymbol{c}^{i}\right)}{p(\boldsymbol{y})}
$$

after applying Bayes' law. $p\left(\boldsymbol{c}^{i}\right)$ is the a priori probability of having the codeword, which here is equal to $1 / \lambda$. As $\boldsymbol{y}$ and $\boldsymbol{c}^{i}$ are vector with $\lambda$ elements, the APP becomes:

$$
p\left(\boldsymbol{c}^{i} \mid \boldsymbol{y}\right)=\frac{1}{\lambda p(\boldsymbol{y})} \prod_{m=0}^{\lambda-1} p\left(y_{m} \mid c_{m}^{i}\right) .
$$

Considering the complex AWGN case,

$$
p\left(y_{m} \mid c_{m}^{i}\right)=\frac{1}{2 \pi \sigma^{2}} \exp \left\{-\frac{1}{2 \sigma^{2}}\left\|y_{m}-c_{m}^{i}\right\|^{2}\right\},
$$

where $\sigma$ is the noise variance and $\|\cdot\|$ is the Euclidean norm. $y_{m}$ and $c_{m}^{i}$ are complex numbers, hence (5) can be expressed 


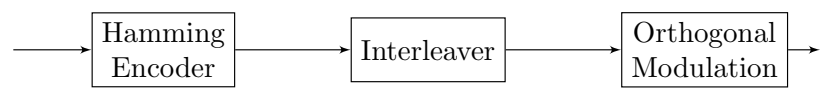

(a)

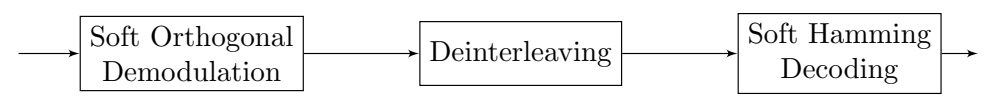

(b)

Fig. 2. Orthogonal modulation and Hamming code: (a) transmitter architecture, (b) receiver architecture.

$$
p\left(\boldsymbol{c}^{i} \mid \boldsymbol{y}\right)=\frac{C}{\lambda p(\boldsymbol{y})} \exp \left\{\frac{1}{\sigma^{2}} \sum_{m=0}^{\lambda-1} \operatorname{Re}\left(y_{m} \cdot \overline{c_{m}^{i}}\right)\right\},
$$

where $C$ is a constant that will be canceled out in further computations, and $\overline{c_{m}^{i}}$ the complex conjugate of $c_{m}^{i}$. LLR of the information bits can be computed using the expression

$$
L\left(d_{n} \mid \boldsymbol{y}\right)=\log \sum_{\substack{i=0, b_{n}^{i}=1}}^{\lambda-1} p\left(\boldsymbol{c}^{i} \mid \boldsymbol{y}\right)-\log \sum_{\substack{i=0, b_{n}^{i}=0}}^{\lambda-1} p\left(\boldsymbol{c}^{i} \mid \boldsymbol{y}\right),
$$

where the index $b_{n}^{i}=1$ (resp. $\left.b_{n}^{i}=0\right)$ for the first sum (resp. the second sum) signifies that the sum is done over the codewords $c^{i}$ that encode an information word for which the value of bit $b_{n}^{i}$ is 1 (resp. 0 ). The factor before the exponential in (7) is suppressed at this step.

Once LLR have been computed, deinterleaving is done. Soft Hamming decoding can then be executed to get the decoded information bits.

\subsection{Standard modulation with turbo coding}

3G and 4G standards both rely on a turbo code for forward error correction [7]. The TC used is a parallel concatenation of two identical recursive systematic codes of rate $1 / 2$, making the code rate equal to $1 / 3$ (or slightly less when the termination bits needed to close the trellis are considered). The constituent code has a constraint length of $k=4$, and its feedback and generator polynomials are respectively [1315] (in octal). This channel code allows any system to achieve an energy efficiency gain relatively high, thanks to iterative soft decoding at the receiver side. Complexity at the transmitter side is rather low. We consider this code combined with a modulation (BPSK or $M$-ary FSK) as a potential physical layer for LPWA network, or at least as an interesting element of comparison.

The architecture of the transmitter is depicted Figure 3. The Parallel-toSerial Conversion has three inputs : the information bits, the parity bits of the first Recursive Systematic Coder (RSC), and the parity bits of the second RSC which input has been interleaved. After serial conversion, a SF of value $\lambda$ is 


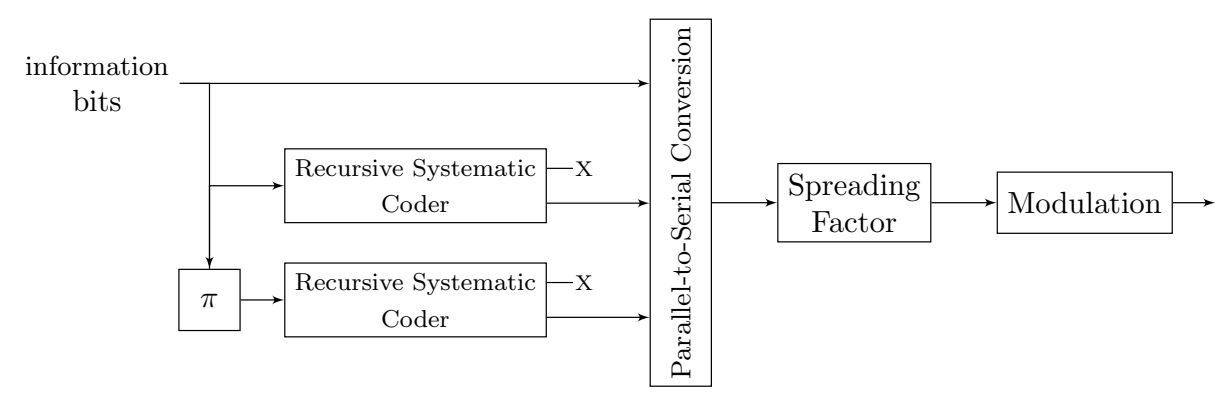

Fig. 3. Modulation and Turbo Code UMTS: transmitter side.

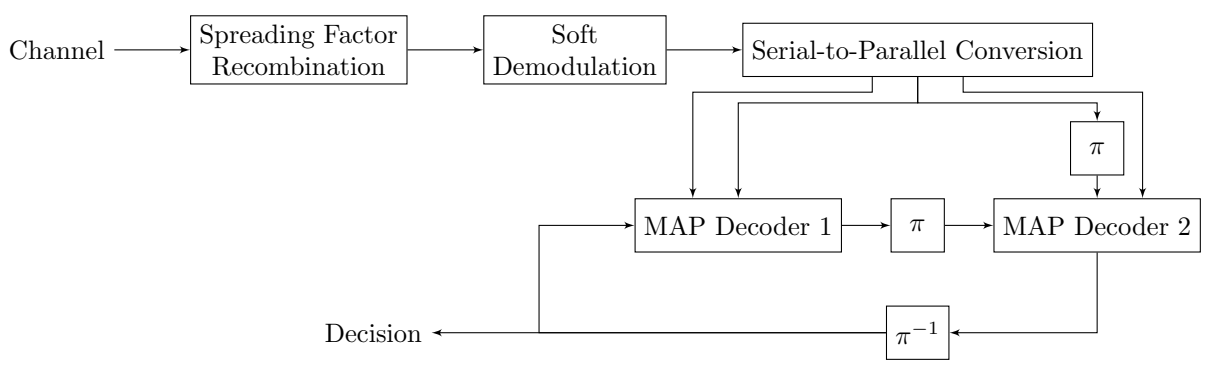

Fig. 4. Modulation and Turbo Code UMTS: receiver side.

applied, and then encoded data is modulated. The spectral efficiency of this physical layer is

$$
\eta_{3}=\frac{\eta_{\mathrm{mod}}}{3 \lambda}
$$

where $\eta_{\text {mod }}$ is the normalized spectral efficiency of the modulation used, defined as

$$
\eta_{\text {mod }}=\left\{\begin{array}{cl}
1 & \text { if BPSK is used, } \\
\frac{\log _{2}(M)}{M} & \text { if } M \text {-ary orthogonal modulation is used. }
\end{array}\right.
$$

The receiver suggested is described Figure 4. After the noisy channel, the SF Recombination module averages $\lambda$ consecutive symbols. Signal is then demodulated, i.e LLR are computed. Serial-to-Parallel Conversion will give the systematic bits (i.e uncoded information bits), and parity bits of each encoder. Each decoder is then fed by the systematic bits (which are interleaved for the second decoder) and parity bits corresponding to each RSC. Maximum A Posteriori (MAP) rule is used to decode the trellis, using the Bahl, Cocke, Jelinek and Raviv (BCJR) algorithm [11]. The max-log approximation can be used, implying a small loss in performance for an interesting reduction of complexity. For each decoder, the output of the other decoder is used as a priori input after interleaving. Several iterations of this process are performed, and a decision can be made at the end of each iteration. 


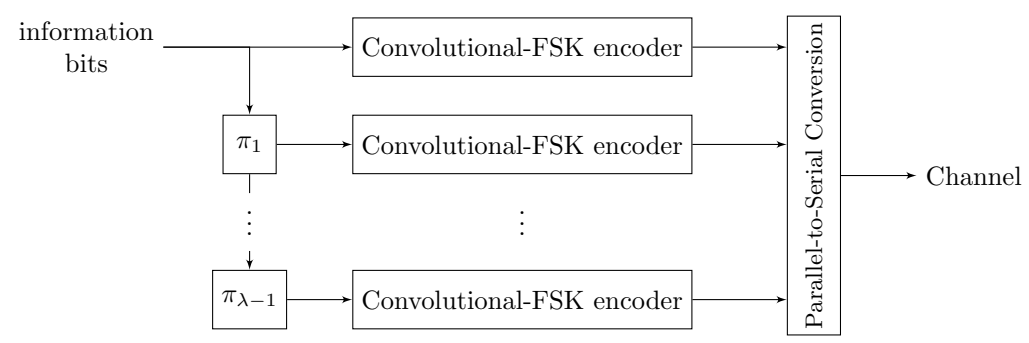

Fig. 5. The Turbo-FSK transmitter.

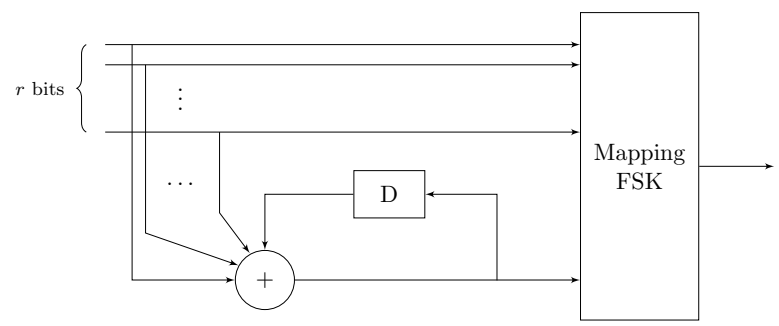

Fig. 6. The Convolutional-FSK encoder.

\subsection{Turbo-FSK}

A recent scheme has been proposed, using orthogonal modulations and turbo decoding at the receiver side [9]. This scheme allows for a better use of redundancy than in pure repetition scheme, and achieves an interesting energy efficiency gain. The transmitter low complexity makes it suitable for uplink communication in the LPWA context.

Transmitter architecture is given in Figure 5. The structure is composed of $\lambda$ stages, each one encoding an interleaved version of the input bits. Information bits are gathered into $P$ groups of $r$ bits, and Convolutional-FSK encoding is applied, as described Figure 6. For every group of $r$ bits, parity is computed and accumulated in the memory. The $r+1$ bits are then mapped to a codeword of the FSK alphabet, which size is $M=2^{r+1}$. Thanks to the accumulator, every consecutive symbol is linked to the previous one. The output of each encoder is then a set of $P$ FSK codewords: this scheme mixes coding and modulation in the same process. A Parallel-to-Serial Conversion is then done to send the FSK codewords through the channel. The spectral efficiency is defined by

$$
\eta_{4}=\frac{\log _{2}(M)-1}{\lambda M},
$$

The receiving side, depicted in Figure 7, consists of Serial-to-Parallel Conversion, to reconstruct the $\lambda$ stages emitted at the first place. A Soft FSK Detector is used to determine the probabilities of each possible codewords. This step can be done using the Fast Fourier Transform (FFT) algorithm. These probabilities are then fed to the APP decoder, which will use them as channel observation, while output 


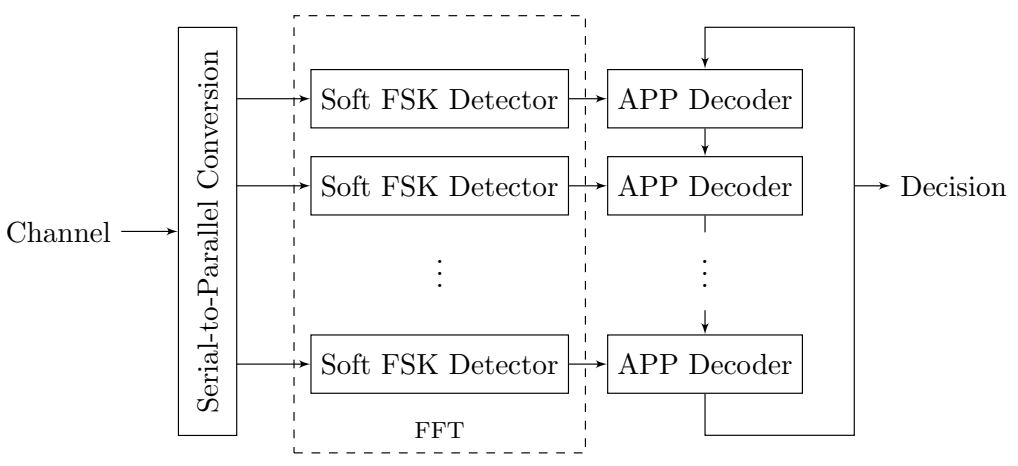

Fig. 7. The Turbo-FSK receiver.

of the other decoders will be used as a priori information. BCJR algorithm is used to decode the trellis. Orthogonality of the transmitted codewords is one of the key feature of this scheme, which offers a great performance gain compared to simple repetition schemes. Detailed explanations about the transmitter and the receiver's computations can be found in [9].

\section{Simulation Results}

To perform a comparison between the schemes presented in Section 2, we perform simulations for the AWGN channel, with coherent reception. Random interleavers are used, except for the TC UMTS where the interleaver specified by the 3G standard is considered. Packet size is set to 1024 bits (or 128 bytes), which is rather small but appropriate for the LPWA context. For iterative schemes, 10 decoding iterations are performed, since performance improvement is rather small for larger numbers of iterations. MAP algorithm is used.

For the sake of fairness, the different schemes should be compared with an equal normalized spectral efficiency $\eta$. This physical feature can be adjusted thanks to the definition of the parameter $\lambda$, which is related to either a repetition factor or an alphabet size. The normalized spectral efficiency of a configuration of the Turbo-FSK (32-FSK with $\lambda=4$ stages, with $\eta_{4} \simeq 1 / 32=3.125 \cdot 10^{-2}$ ) is taken as reference. The adjusted spectral efficiencies, the parameters $\lambda$, the modulations, the FEC chosen and the binary code rate are summarized in Table 1. For the third scheme, two different modulations are chosen: BPSK (physical layer 3) and 32-FSK (physical layer 3bis), hence the total of 5 physical layers. To obtain the SNR, we consider the straightforward conversion

$$
\mathrm{SNR}=\eta \frac{E_{b}}{N_{0}}
$$

Figure 8 shows the Bit Error Rate (BER) performance versus SNR for the 5 physical layers. All schemes have interesting performance at a very low SNR, and will work at low sensitivity levels, which was the goal to achieve. Scheme 


\begin{tabular}{ccccccc} 
PHY-layer & 1 & 2 & 3 & 3 bis & 4 \\
\hline Modulation & DBPSK & 128-Orthog & BPSK & 32-FSK & 32-FSK \\
\hline FEC & CC [171 133] & Hamming & TC [13 15] & TC [13 15] & Turbo-FSK \\
\hline $\begin{array}{c}\text { Binary } \\
\text { code-rate }\end{array}$ & $1 / 2$ & $4 / 7$ & $1 / 3$ & $1 / 3$ & - \\
\hline$\lambda$ & 16 & 128 & 11 & 2 & 4 \\
\hline$\eta\left(\cdot 10^{-2}\right)$ & 3.125 & 3.120 & 3.019 & 2.594 & 3.113 \\
\hline
\end{tabular}

Table 1. Parameters used for comparison. Termination bits for convolutional codes are considered when computing the normalized spectral efficiency values, implying a lower value than expected. For Turbo-FSK, no binary code rate can be defined, since coding and modulation are mixed.

1 shows the typical performance of a convolutional code. Scheme 2 uses the Hamming code, which is less powerful than the convolutional code of scheme 1 , but offers better performance when combined with a relatively large size of alphabet (128). Performance clearly shows the gain of turbo processing. Scheme 3 , corresponding to the Turbo Code UMTS with BPSK modulation and $\lambda=11$, achieves a BER of $10^{-5}$ for a SNR of $-14.2 \mathrm{~dB}$, hence outperforming scheme 2 by $4.5 \mathrm{~dB}$. Scheme $3 \mathrm{bis}$, for which 32-FSK modulation is used, suffers from a performance loss compared to 3 . Scheme 4 outperforms all the other schemes, showing the beneficial impact of mixing convolutional encoding and FSK modulation as proposed in [9]. It should be noted that both schemes 3bis and 4 use the same size of orthogonal alphabet, with yet a gain greater than $1.3 \mathrm{~dB}$ at a BER of $10^{-4}$ for the Turbo-FSK scheme.

Packet Error Rate (PER) performance versus the SNR is depicted Figure 9. Schemes involving turbo processing are again shown to be far more efficient than the other schemes, with a gain superior to $3.5 \mathrm{~dB}$ for a PER of $10^{-3}$.

The ultimate Shannon's limit [12] is the maximal transmission rate with arbitrarily small bit-error probability, for a given SNR and a given bandwidth. Comparing schemes to this limit thus shows how efficiently the channel resource is used. The limit, expressed in terms of normalized spectral efficiency versus $E_{b} / N_{0}$, is represented in Figure 10, along with the performance of the 5 physical layers that are here presented. $M$-ary orthogonal modulation is also added for reference. Since we choose a specific value of normalized spectral efficiency, all performance corresponding to the schemes are approximately on the same horizontal line. Scheme 4 is the closest scheme to the $E_{b} / N_{0}$ Shannon's limit as it is only $2.3 \mathrm{~dB}$ away, showing it to be the scheme with the best energy efficiency. We also give the performance of scheme 3 but without any repetition (thus $\lambda=1$ ). As repetition does not influence performance in $E_{b} / N_{0}$, both schemes have the same energy efficiency (same vertical line). Comparing scheme 4 to the uncoded $M$-ary orthogonal modulation, performance shows that turbo processing is a 


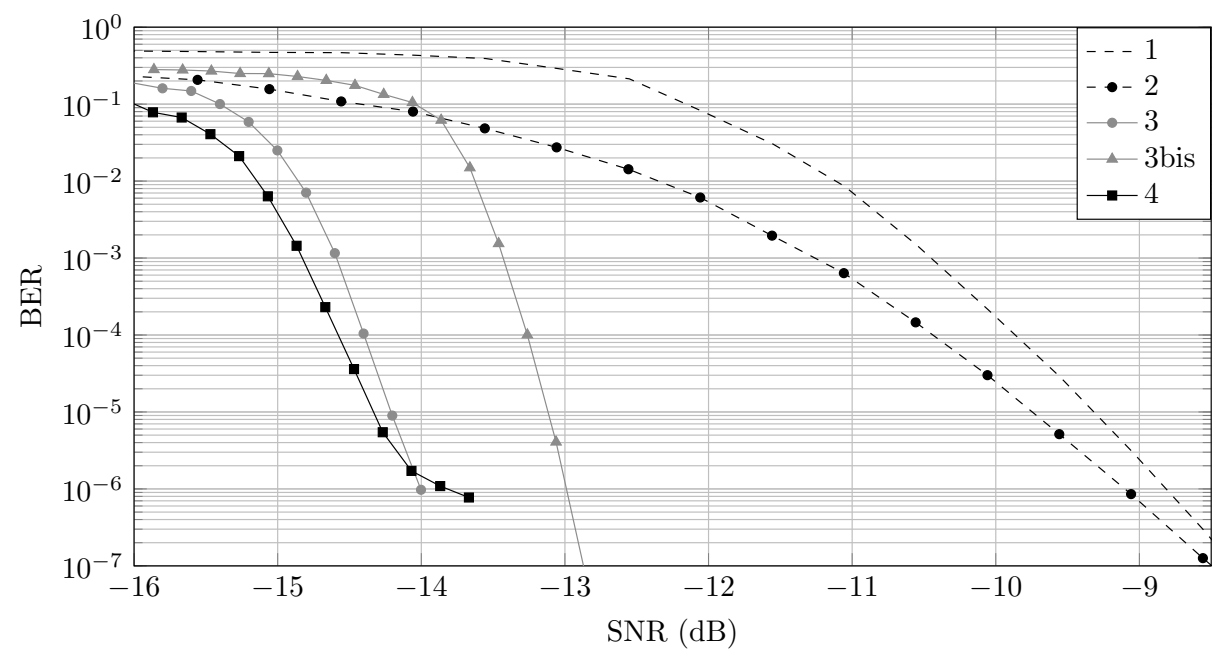

Fig. 8. BER performance comparison in SNR. For schemes using turbo processing (plain curves), 10 iterations have been performed and the block size is set to 1024 bits. Spectral efficiency is roughly equal to $3.113 \cdot 10^{-2} \mathrm{bits} / \mathrm{s} / \mathrm{Hz}$.

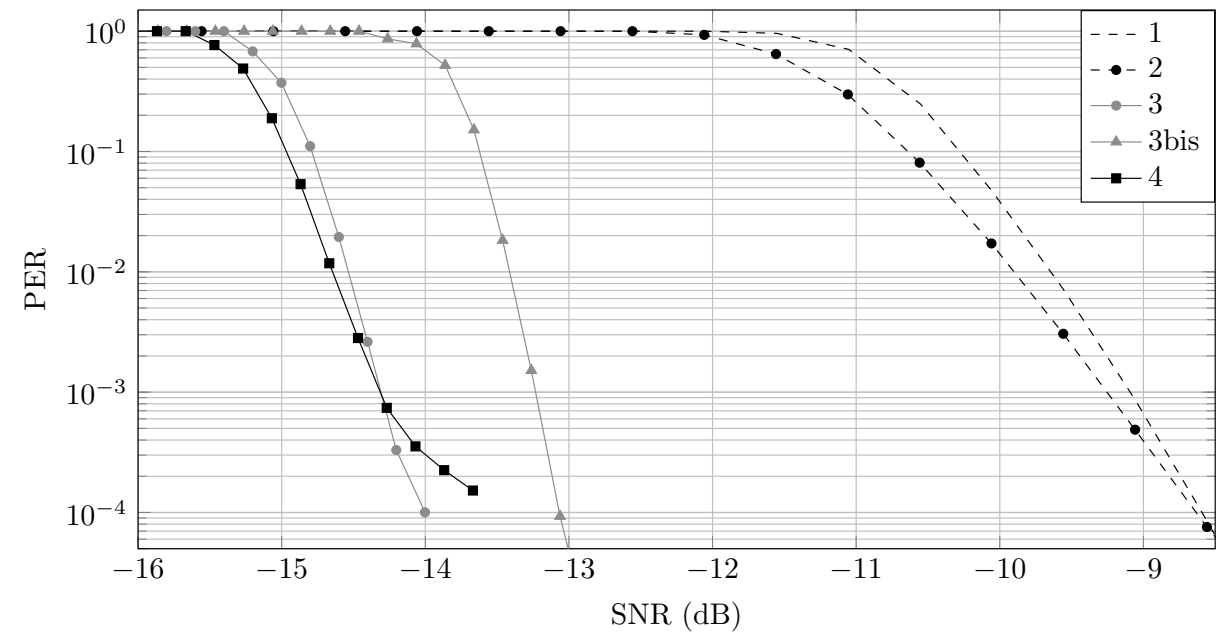

Fig. 9. PER performance comparison in SNR. Packet size is 1024 bits. For schemes using turbo processing (plain curves), 10 iterations have been performed and the block size is set to 1024 bits. Spectral efficiency is roughly equal to $3.113 \cdot 10^{-2} \mathrm{bits} / \mathrm{s} / \mathrm{Hz}$.

way to get closer to Shannon's limit with a reasonable size of alphabet, hence a reasonably low spectral efficiency. 


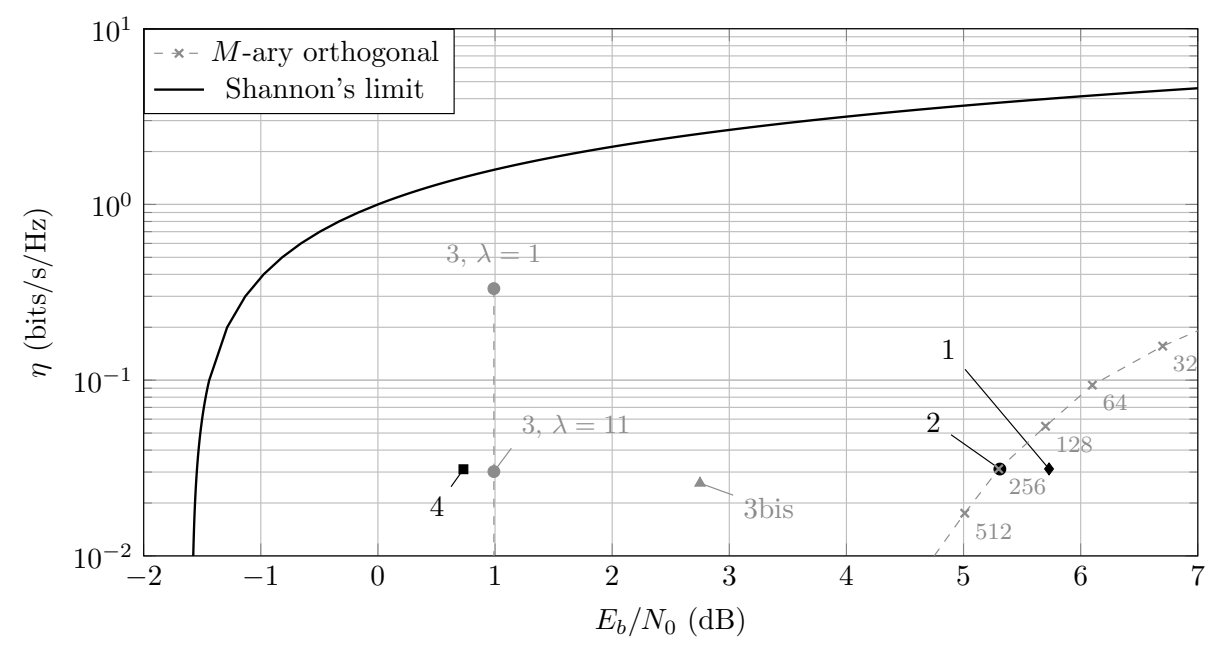

Fig. 10. Performance comparison considering spectral efficiency versus $E_{b} / N_{0}$. The given $E_{b} / N_{0}$ values are the ones for which the BER is $10^{-5}$. For schemes using turbo processing, 10 iterations have been performed and the block size is set to 1024 bits.

\section{Discussion}

As presented in Section 3, schemes involving turbo processing have clearly the potential to reach low sensitivities. All gains presented in the previous section can be associated to the gain in sensitivity: using scheme 4 instead of scheme 1 with the same bandwidth will lead to the same data rate but sensitivity will be $5 \mathrm{~dB}$ lower. Same performance can thus be achieved with a transmitted power divided by 3.1 (in linear scale) or distance between transmitter and receiver increased by a factor 1.7 (considering a free space path loss). In an other way, for a same level of sensitivity, schemes based on repetition will have to use a higher $\mathrm{SF}$, thus a longer signal for an equivalent bandwidth. This can imply harder constraints for synchronization, in terms of phase noise or channel coherence time.

The performance improvements are done at the expense of an increased complexity. However, the cost of complexity of the turbo processing-based schemes is paid by the receiver side, i.e the base station, which can be considered to have unlimited resource. This is not often suggested for the LPWA context, but more advanced processing can be considered, leading to better performance.

As mentioned in Section 1, we assumed perfect synchronization. Since every communication system needs to be synchronized to work properly, this aspect must be taken into account. This is one of the major issues concerning low SNR communication, that still needs to be solved. 


\section{Conclusion}

The design of a new LPWA network is a major issue addressed in the context of the IoT. The uplink scheme is critical, as terminals must have low power consumption and high reliability. We compared several schemes as potential candidates, and have shown that if repeating the information is a good way to lower the sensitivity, more complex systems can allow for higher energy efficiency gain. Simulation results show that turbo processing is an efficient way to achieve a gain in sensitivity. Amongst the studied turbo schemes, Turbo-FSK is the most efficient, and demonstrates the positive impact of mixing turbo processing, $M$ ary orthogonal modulation and low spectral efficiency. However, synchronization for this ranges of SNR and complexity remain open questions.

\section{References}

1. T. Rebbeck, M. Mackenzie, and N. Afonso, "Low-powered wireless solutions have the potential to increase the M2M market by over 3 billion connections," Analysys Mason, Sept 2014.

2. J. Proakis, Digital Communications 3rd Edition, ser. Communications and signal processing. McGraw-Hill, 1995.

3. "LoRa Alliance," https://www.lora-alliance.org/, accessed: February 29, 2016.

4. O. Seller and N. Sornin, "Low power long range transmitter," US Patent 20140219329 A1, Aug 2014.

5. "802.15.4k: Low-Rate Wireless Personal Area Networks (LR-WPANs) Amendment 5: Physical Layer Specifications for Low Energy, Critical Infrastructure Monitoring Networks." IEEE Standard for Local and metropolitan area networks, pp. 1-149, Aug 2013.

6. C. Berrou, A. Glavieux, and P. Thitimajshima, "Near Shannon limit error-correcting coding and decoding: Turbo-codes. 1," in IEEE International Conference on Communications (ICC). Geneva., vol. 2, May 1993, pp. 1064-1070.

7. "LTE Evolved Universal Terrestrial Radio Access (E-UTRA): Multiplexing and Channel Coding," 3GPP TS 36.212, V12.6.0, Release 12, pp. 12-15, Oct 2015.

8. M. C. Valenti and J. Sun, "The UMTS Turbo Code and an Efficient Decoder Implementation Suitable for Software Defined Radios," International Journal of Wireless Information Networks, vol. 8, pp. 203-216, 2001.

9. Y. Roth, J.-B. Dore, L. Ros, and V. Berg, "Turbo-FSK: A New Uplink Scheme for Low Power Wide Area Networks," in 2015 IEEE 16th International Workshop on Signal Processing Advances in Wireless Communications (SPAWC), June 2015, pp. $81-85$.

10. "SX1272 from Semtech, datasheet," http://www.semtech.com/wireless-rf/ rf-transceivers/sx1272/, accessed: February 29, 2016.

11. L. Bahl, J. Cocke, F. Jelinek, and J. Raviv, "Optimal decoding of linear codes for minimizing symbol error rate (corresp.)," IEEE Transactions on Information Theory, vol. 20, no. 2, pp. 284-287, Mar 1974.

12. C. Shannon, "A mathematical theory of communication," The Bell System Technical Journal, vol. 27, no. 3, pp. 379-423, July 1948. 